\title{
ARTÍCULO ORIGINAL \\ Diagnóstico clínico de alotrofagia en bovinos del departamento de Sucre Colombia
}

\author{
Clinical diagnosis of allotrophagia in cattle of the department of Sucre \\ Colombia
}

\author{
Cardona A José ${ }^{1}$ Ph.D; Montes V Donicer² Ph.D; Martínez M Mastoby³ M.Sc. \\ ${ }^{1}$ Universidad de Córdoba, Facultad de Medicina Veterinaria y Zootecnia, Grupo de \\ Investigación en Medicina de Grandes Animales (MEGA), Montería, Colombia. \\ ${ }^{2}$ Universidad de Sucre. Facultad de Ciencias Agropecuarias, Departamento de Zootecnia. \\ Grupo de Investigación en Mejoramiento y Reproducción Animal Sincelejo, Colombia. \\ ${ }^{3}$ Universidad de Córdoba, Facultad de Medicina Veterinaria y Zootecnia. Montería, Colombia.
}

\section{Keywords:}

Depraved appetite;

pica;

minerals;

deficiency.

\begin{abstract}
The objective of this study was to analyze and describe the clinical characteristics of 17 cases of allotrophagia in calves of both sexes and ages between 14 and 25 months of age from the municipality of the union, Sucre, Colombia, between January And April in the period from 2005 to 2016. A general clinical and special digestive examination was performed, with similar clinical manifestations in all animals, which consisted of evidence of poor body condition, anemia and edema in the jowl, as well as increased volume in the lower left quadrant of the abdomen, when performing movements of succussion and percussion of the abdomen was evidenced the presence of solid material in great quantities that emitted a sound similar to the blow of several stones in a sac and to the auscultation it was found fading of the ruminal sounds. All animals underwent surgical removal of the material by rumenotomy, mainly finding stones, plastic, bones and seeds of Elaeis oleifera (corozo). The diagnosis of allotrophagia was made based on anamnesis, the clinical characteristics in the animal and the surgical extraction of the material, which are characteristic in this type of nutritional alteration. Although it is a common event in cattle from this region of Sucre department, this is the first report of allotrophagia in cattle of this department and Colombia.
\end{abstract}

\section{Resumen}

El objetivo de este estudio fue analizar y describir las características clínicas de 17 casos de alotrofagia en terneros, de ambos sexos y edades comprendidas entre 14 y 25 meses de edad, procedentes del municipio de la unión, Sucre (Colombia) entre los meses de enero y abril en el periodo de 2005 al 2016. Se realizó examen clínico general y digestivo especial encontrándose manifestaciones clínicas similares en todos animales, las cuales consistieron en la evidencia de mala condición corporal, anemia y edema en la papada, así como aumento de volumen en el cuadrante inferior izquierdo del abdomen, al realizar movimientos de sucusión y percusión del abdomen se evidencio la presencia de material sólido en grandes cantidades que emitían un sonido parecido al golpe de varias piedras en un saco y a la auscultación se encontró apagamiento de los sonidos ruminales. A todos los animales se les realizó extracción quirúrgica del material mediante ruminotomía, encontrándose principalmente piedras, plástico, huesos y semillas de Elaeis oleífera (corozo). El diagnóstico de alotrofagia se realizó basado en la anamnesis, las características clínicas en el animal y la extracción quirúrgica del material, que son características en este tipo de alteración nutricional. A pesar de que es evento común en bovinos provenientes esta región del departamento de Sucre, este es el primer reporte de alotrofagia en bovinos de este departamento y Colombia. 


\section{Introducción}

La alotrofagia (del griego "allótrios": extraño, y "phagos": comer) (MARTINS et al., 2004), es un desorden o perversión del apetito, caracterizado por la ingesta persistente y en ocasiones compulsiva de materiales usualmente no comestibles, como como tierra (geofagia), pelos (tricofagia), piedras (litofagia), madera, (lignofagia), y huesos (osteofagia), entre otros (FIESTAS-TEQUE, 2014; ROSSANIGO et al., 2014). Este trastorno también recibe el nombre de cissa, picacia, pellacia, malacia, pseudorexia, parorexia, geomanía, y pica (CAMPUZANO, 2011), este último es uno de los más utilizados, y es tomado del latín "pica", palabra utilizada para nombrar a la urraca (Pica pica) ave de la familia corvidae que es conocida por robar y consumir de forma indiscriminada todo tipo de objetos no alimenticios (FIRYAL, 2007; CAMPUZANO, 2011; FIESTAS-TEQUE, 2014).

La principal causa de la pica es la deficiencia de macro minerales esenciales como Calcio $(\mathrm{Ca})$, Fosforo $(\mathrm{P})$, Magnesio (Mg), y micro minerales como Hierro (Fe), Cobre (Cu), Manganeso (Mn), Selenio (Se), Zinc (Zn) y Cobalto (Co); especialmente el fosforo (OCAL et al., 2008).

Se ha descrito la relación de la alotrofagia con la etapa productiva, siendo más común en animales jóvenes, pudiendo también encontrarse tricobenzoares (masas ligeras, suaves o peludas, formadas por pelo ingerido plegado, mezclado con alimento y fibra, de color verdoso oscuro a marrón negro, con olor fétido por la fermentación y descomposición de alimentos intercalados con resto orgánicos), probablemente por las continuas privaciones de sodio $(\mathrm{Na})$ en este tipo de animales, por lo que adquieren el hábito de lamerse el sudor unos a otros como intento de suplir este requerimiento, mientras que en los adultos es más común encontrar la presencia de huesos, incluyendo dientes; piedras de diferente tamaños y formas, virutas de madera, corteza de árbol, pequeñas ramas; fragmentos de vidrio, objetos metálicos como clavos, alambres y fragmentos de estaño; bolsas, fragmentos de plásticos, recipientes de comida, arena, cuero, entre otros (MARTINS et al., 2004; FIRYAL, 2007; OCAL et al., 2008; SIMSEK et al., 2015).

Las deficiencias de minerales se presentan con signos inespecíficos como, despigmentación del pelo y piel, pérdida de peso, mucosas pálidas, articulaciones endurecidas o engrosadas, nacimientos de crías débiles, pérdida de la libido, repetición o ausencia de celos, abortos no infecciosos, muerte súbita, alteraciones óseas o dentarias, baja natalidad, inmunodepresión, diarrea, anemia, osteodistrofia, hipocalcemia, disminución en la producción de leche, hirsutismo y pica (OCAL et al., 2008; OLIVEIRA et al., 2010; LOPES et al., 2013).

De forma general, los cuerpos extraños son retenidos en el rumen o retículo, pudiendo moverse inter reticularmente, materiales con característica puntiaguda (como huesos, clavos, vidrios, etc.) pueden atravesar la pared del estómago y perforar el pericardio (retículopericarditis), pleura, pulmón izquierdo, hígado o bazo, ocasionando complicaciones graves, por lo que se hace necesario descartar compromiso de otros órganos, para el caso de la reticulopericarditis, se recomienda evaluar la presencia de dolor reticular, haciendo la prueba de "Gotze", también conocida como prueba de pellizcamiento, prueba del bastón o prueba de presión reticular a nivel del esternón (MARTINS et al., 2004).

Para el caso de la ingesta de huesos (osteofagia), se ha reportado como un factor predisponente para la ocurrencia de botulismo, enfermedad infecciosa causada por el Clostridium botulinum, el cual crece en cadáveres y material orgánico en descomposición, y a medida que el microorganismo crece produce una potente neurotóxina que causa parálisis y los animales mueren por paro cardiorrespiratorio (BENAVIDES et al., 2011).

La ingesta de bolsas de plástico o fragmentos de este, comúnmente ocasiona obstrucciones del píloro, seguido de una distención abdominal a nivel de la fosa del ijar izquierda, por inhibición del reflejo del eructo, acumulación de gas y consecuente timpanismo, de igual forma, el acumulo de materiales pequeños y compactos como pequeñas piedras, grava y arena en rumen, predispondrán a impacciones, en los que se evidencia una distención abdominal en la zona ventral izquierda, siendo más común en animales con cama de arena, o en animales que consumen agua con altos contenidos de arena de forma prolongada (MARTINS et al., 2004; GONZÁLEZ y VALENCIA, 2011; SIMSEK et al., 2015). Se ha reportado en plantas de sacrificio la presencia de úlceras abomasales de forma concomitante con cuerpos extraños, presuntamente por acción mecánica de estos últimos (HUND et al., 2016).

El diagnostico de cuerpo extraños se hace teniendo en cuenta los antecedentes de pica o alotrofagia, sumado a presencia de uno o más signos clínicos característicos de deficiencia de minerales, presencia de complicaciones dependientes a la naturaleza y cantidad de los materiales consumidos, descarte de peritonitis y compromiso de otros órganos, laparotomía y posterior ruminotomia exploratoria; por otra parte se ha plateado el uso de detectores de minas terrestres como ayuda en la investigación de cuerpo extraños metálicos en bovinos con sintomatología de gastritis traumática, pero su utilidad es cuestionada por la 
poca disponibilidad del equipo (MARTINS et al., 2004; GONZÁLEZ y VALENCIA, 2011).

Debido a que la Alotrofagia es un signo y no enfermedad, el tratamiento va dirigido al hallazgos de cuerpo extraños, que dependiendo de la gravedad del caso consiste en extracción por ruminotomia; y la corrección de la deficiencia de minerales en la dieta, sin embargo, en casos graves con compromiso de otros órganos se ha sugerido la eutanasia (ROSSANIGO et al., 2014; GONZÁLEZ y VALENCIA, 2011). El objetivo de este estudio fue analizar y describir las características clínicas de la alotrofagia en terneros del departamento de Sucre (Colombia).

\section{Materiales y métodos}

17 terneros mestizos, manejados bajo el sistema doble propósito, de ambos sexos (11 machos y 6 hembras), con edades comprendidas entre 14 y 25 meses de edad y pesos promedio de $220 \pm 20$ kilos, procedentes del municipio de la Unión (Sucre, Colombia), fueron atendidos en el servicio clínico ambulatorio de grandes animales de la Facultad de Medicina Veterinaria y Zootecnia de la Universidad de Córdoba entre los meses de enero y abril en un periodo comprendido entre el 2005 y 2016.

A los animales del estudio, se les realizó examen clínico general y digestivo especializado, consistente en evaluación clínica del paciente (condición corporal, características de las mucosas, tiempo de llenado capilar, pulso, frecuencias cardiaca y respiratoria) y del aparato digestivo (inspección, palpación, percusión y auscultación), con énfasis en el abdomen (forma, tensión, sonido percutorio, intensidad de los sonidos digestivos). Todos los animales presentaron asimetría abdominal con aumento en el cuadrante inferior izquierdo (Figura 1), que al examen clínico del abdomen mediante la prueba de sucusión y percusión (Figura 2) evidenció un sonido característico al de piedras contenidas en un recipiente, por lo que fueron denominadas como "vacas maracas", por su parecido en el sonido que producen estos instrumentos.

Posteriormente, previa sedación (Acepromacina 1\%, Zoo®, Colombia) y aplicación de anestesia local (Lidocaina $2 \%$, Synthesis $₫$, Colombia), se procedió a la extracción del material mediante la técnica quirúrgica de ruminotomía paralumbar izquierda (MUZZI et al., 2013) (Figura 3), en la cual se extrajeron los materiales contenidos en la cavidad ruminal de los animales del estudio, siendo en la mayoría de los casos piedras, plástico, semillas de Elaeis oleifera (corozo) y huesos (Figura 4), siendo pesadas, caracterizadas y fotografiadas (Sony DSC-HV10, China) para su posterior análisis.

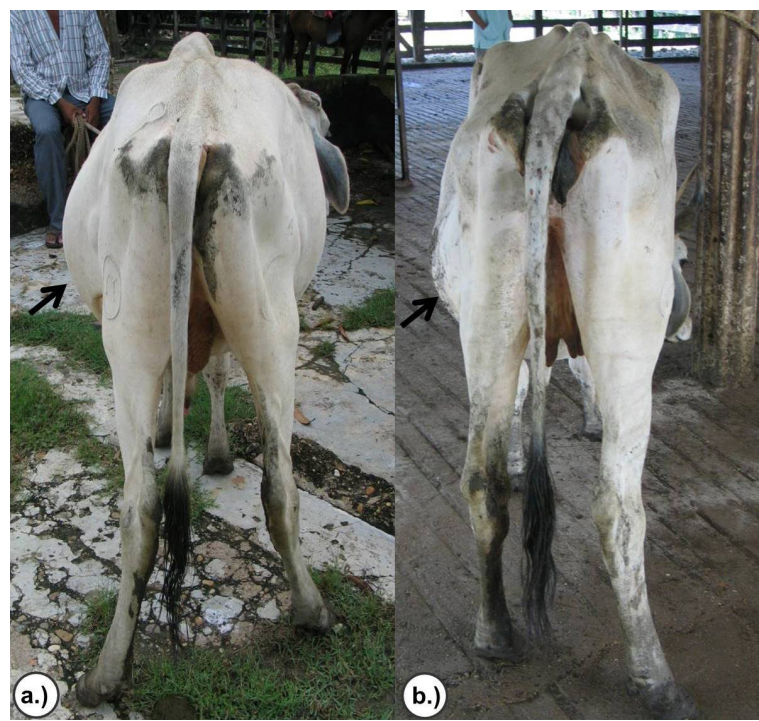

Figura 1. Animales con alotrofagia. Observe la asimetría abdominal con aumento en el cuadrante inferior izquierdo (flechas).

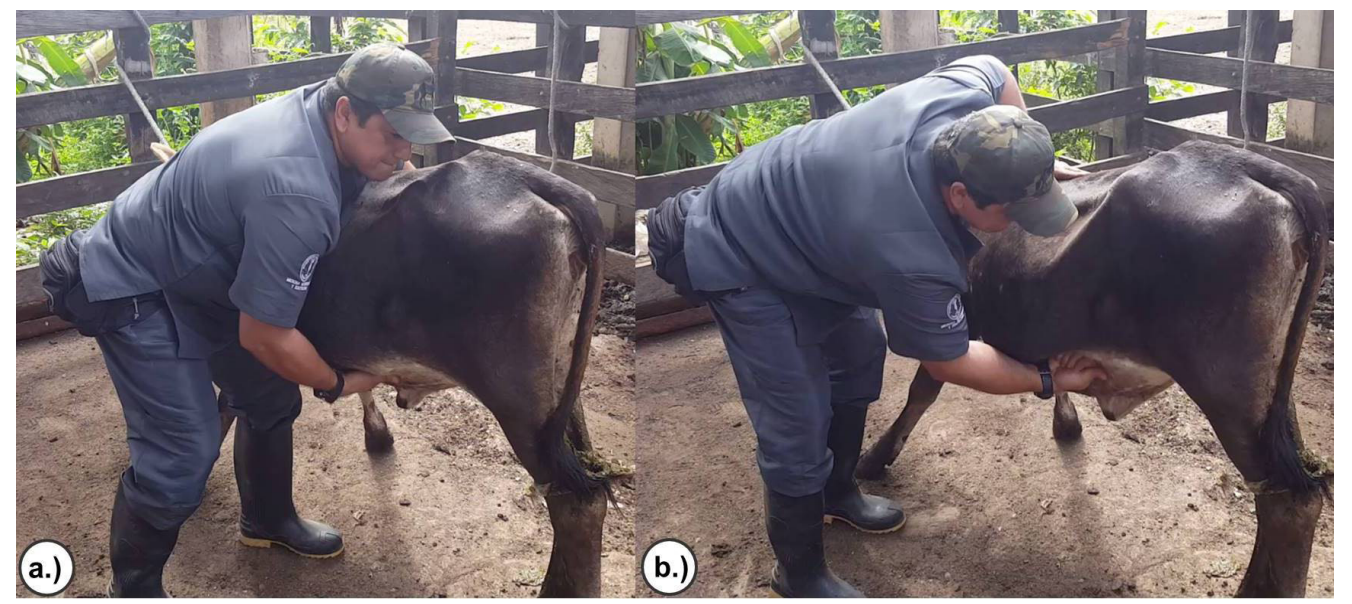

Figura 2. Examen clínico del abdomen mediante la prueba de sucusión (a) y percusión (b) evidenciando un sonido característico al de piedras contenidas en un recipiente, por lo que fueron denominadas como "vacas maracas". 


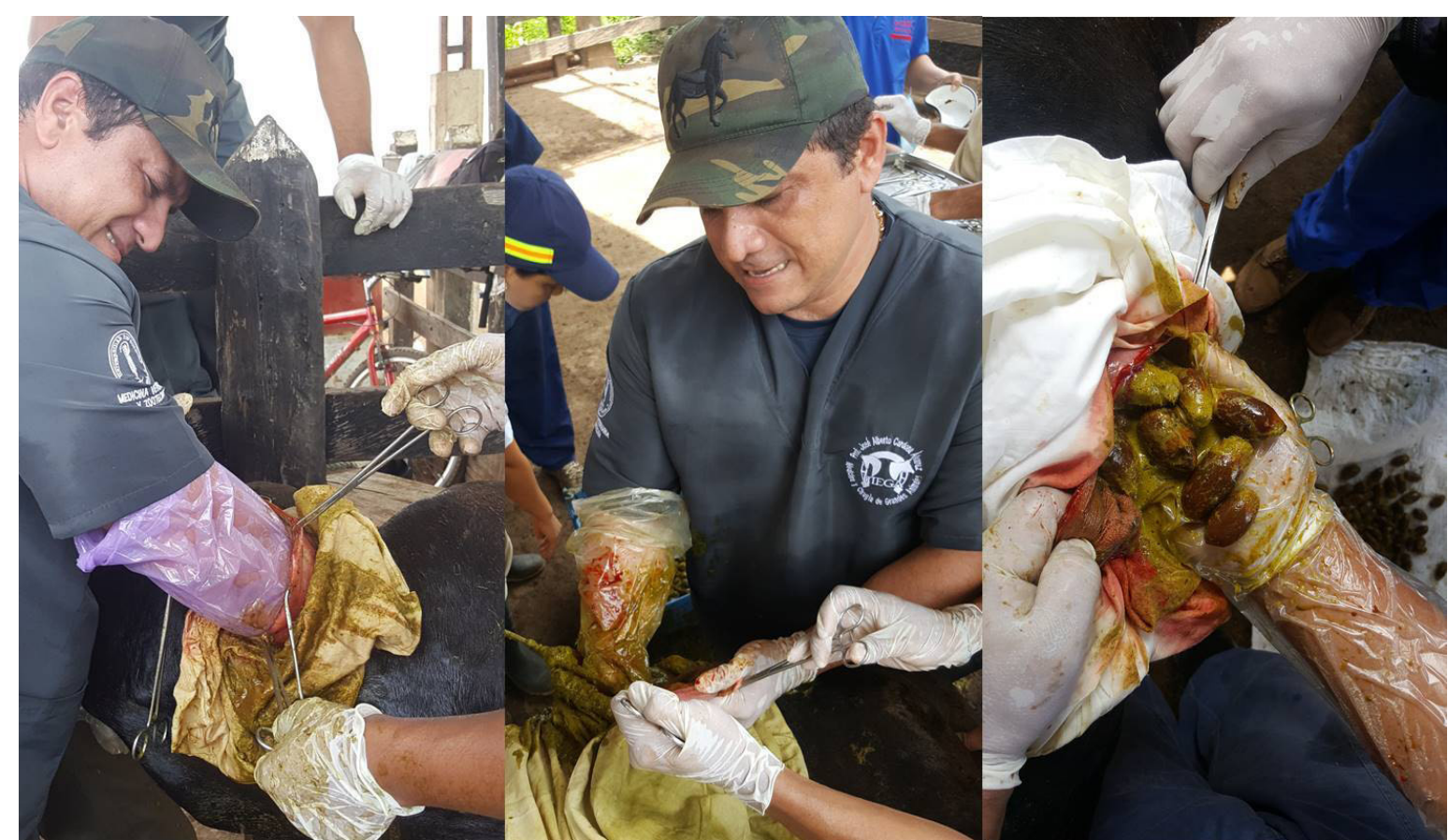

Figura 3. Extracción del material mediante la técnica quirúrgica de ruminotomía paralumbar izquierda.

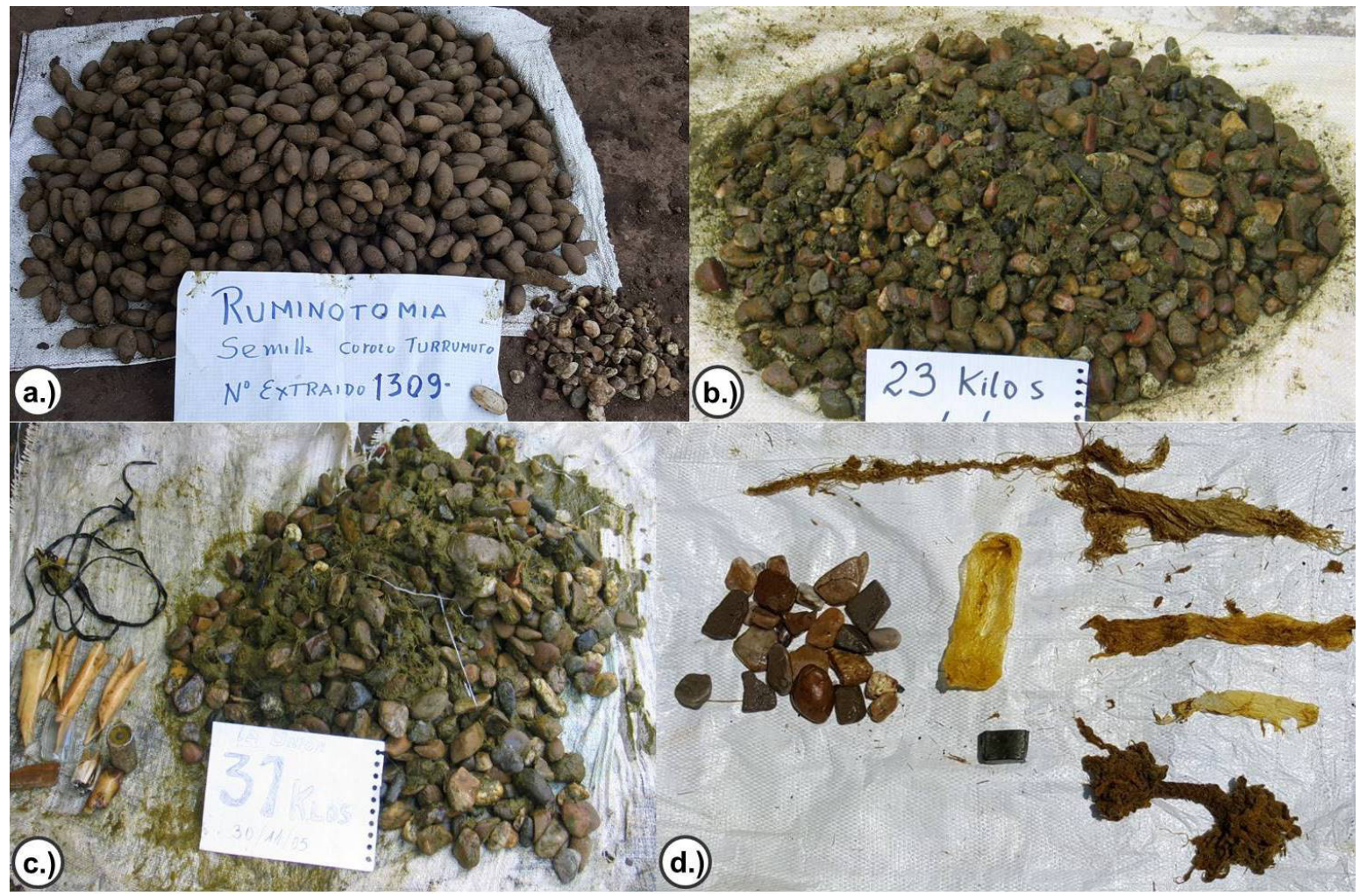

Figura 4. Materiales extraídos de la cavidad ruminal de los animales del estudio Observe la presencia de: a.) 1309 semillas de Elaeis oleifera (corozo) y piedras. b.) 23 kilos de piedras. c.) huesos, pitas, baterías y 31 kilos de piedras y d.) piedras, botella y bolsas plásticas y sogas. 


\section{Resultados y discusión}

No existen reportes en la literatura consultada sobre las características clínicas de alotrofagia en bovinos del departamento de Sucre y Colombia.

Los datos de la anamnesis como la procedencia de una zona deficiente en minerales y en época de sequía, así como las manifestaciones obtenidas en la exploración física como debilidad, pelaje hirsuto, pérdida de peso, mucosas pálidas, asimetría del abdomen con aumento en el cuadrante inferior izquierdo, el sonido de maracas a la sucusión y percusión y el apagamiento de los sonidos digestivos bovinos confirmaron el diagnóstico de alotrofagia en todos los bovinos estudiados, de acuerdo con lo reportado como método diagnóstico de la condición patológica (OCAL et al., 2008; OLIVEIRA et al., 2010; GONZÁLEZ y VALENCIA, 2011; LOPES et al., 2013).

En la Tabla 1, se discriminan los casos de los terneros procedentes del municipio de la unión, Sucre Colombia, que presentaron alotrofagia diagnosticada clínicamente y corroborado por ruminotomía exploratoria. De los materiales extraídos, la mayor presentación fue la litofagia (piedras) en el 52,9\% (9/17), seguido de plasticofagia (plástico) con $29,4 \%(5 / 17)$, semilla de Elaeis oleifera (corozo) con $11,8 \%(2 / 17)$ y osteofagia (hueso) en el 5,9\% (1/17) respectivamente.

El municipio de la Unión, en el departamento de sucre, se caracteriza por poseer deficiencias nutricionales de sus suelos y pasturas, coincidiendo con BENAVIDES (2004) y BENAVIDES et al. (2011), quienes expresan que los suelos de las sabanas tropicales de América latina en su mayoría corresponden a oxisoles, suelos ácidos ( $\mathrm{pH}: 5.0-5.5)$, ricos en óxidos de hierro y aluminio que poseen dificultades de intercambio catiónico, siendo el fósforo el elemento más deficiente; así mismo, OCAL et al. (2008), indican que la principal causa de la pica es la deficiencia de macro minerales esenciales como Calcio (Ca), Fosforo (P), Magnesio (Mg), y micro minerales como Hierro (Fe), Cobre (Cu), Manganeso $(\mathrm{Mn})$, Selenio (Se), Zinc (Zn) y Cobalto (Co).

Los animales fueron atendidos durante los meses de enero a abril, que coincide con las épocas de sequía en la costa norte de Colombia, es así como MARTINS et al. (2004) y MALAFAIA et al. (2011), manifiestan que las deficiencias minerales también se ven reflejadas en la pastura y consecuentemente terminan afectando a la especie bovina; condiciones ambientales que sumadas a la baja sensibilidad de sus órganos gustativos (lengua y labios), la avidez de los animales por sustancias con contenido de $\mathrm{P}$ y $\mathrm{Ca}$, y el hábito de masticar rápidamente los alimentos, predisponen al desarrollo de aberraciones del apetito, en los que se cree, el animal se encuentra en una constante búsqueda no selectiva de los sustancias, y consecuentemente a ingerir una gran cantidad y variedad de cuerpos extraños.

Las edades de los animales oscilaron entre los 14 y 25 meses, coincidiendo con lo reportado por FIRYAL, (2007) y OCAL et al. (2008), quienes indican que se ha descrito la relación de la alotrofagia con la etapa productiva, siendo común los reportes en animales jóvenes, preñados y el primer periodo de lactancia, ya que se cree que esto es debido a que en estas etapas, los requerimientos de minerales en la dieta se ven aumentados incluso 3 veces más de lo normal.

Tabla 1. Descripción de alotrofagia según sexo, edad y material extraído por ruminotomía en 17 terneros mestizos del departamento de Sucre, Colombia.

\begin{tabular}{|c|c|c|c|c|c|}
\hline $\mathbf{N}^{\circ}$ & Raza & Edad (meses) & Sexo & Peso $(\mathrm{Kg})$ & Material extraído \\
\hline 1 & Mestizo & 14 & Macho & 120 & Piedras \\
\hline 2 & Mestizo & 15 & Macho & 130 & Piedras \\
\hline 3 & Mestizo & 17 & Macho & 155 & Piedras \\
\hline 4 & Mestizo & 17 & Macho & 160 & Piedras \\
\hline 5 & Mestizo & 18 & Macho & 170 & Plástico (bolsas) \\
\hline 6 & Mestizo & 18 & Macho & 170 & Semilla de Elaeis oleifera \\
\hline 7 & Mestizo & 19 & Macho & 190 & Piedras, tela de jean \\
\hline 8 & Mestizo & 19 & Macho & 210 & Plástico (botella de agua) \\
\hline 9 & Mestizo & 22 & Macho & 230 & Plástico (bolsas) \\
\hline 10 & Mestizo & 23 & Macho & 230 & Plástico (bolsas y botellas) \\
\hline 11 & Mestizo & 25 & Macho & 240 & Huesos, batería, piedras \\
\hline 12 & Mestizo & 17 & Hembra & 150 & Piedras, pitas \\
\hline 13 & Mestizo & 19 & Hembra & 170 & Piedras \\
\hline 14 & Mestizo & 20 & Hembra & 180 & Piedras \\
\hline 15 & Mestizo & 20 & Hembra & 190 & Piedras, sogas \\
\hline 16 & Mestizo & 22 & Hembra & 190 & Plástico (guantes de palpar) \\
\hline 17 & Mestizo & 24 & Hembra & 200 & Semilla de Elaeis oleifera \\
\hline
\end{tabular}


De igual forma, MARTINS (2004), indica que en animales jóvenes se suele encontrar tricobenzoares, probablemente por las continuas privaciones de sodio ( $\mathrm{Na}$ ) en este tipo de animales, por lo que adquieren el hábito de lamerse el sudor unos a otros como intento de suplir este requerimiento. Por otra parte, SIMSEK et al. (2015), expresan que hallazgo de huesos, incluyendo dientes; piedras de diferente tamaños y formas, objetos metálicos como clavos, alambres y fragmentos de plásticos, recipientes de comida, son más comunes en animales mayores, como fue encontrado en los animales más viejos del presente estudio.
Consideraciones finales. La alotrofagia, se presenta en mayor frecuencia en animales con dietas alimenticias mal balanceada, generalmente, con limitaciones en el suministro de minerales. En las regiones tropicales, los forrajes normalmente no atienden todos los minerales en cantidades suficientes para la producción animal, siendo importante la suplementación mineral de los bovinos a pasto de manera racional y lógica, basada en las exigencias de cada categoría de la estructura del hato, en el diagnóstico de deficiencias clínicas y sub-clínicas de cada animal y a través de la ensayos experimentales. La mejor forma de prevenir desordenes metabólicos es haciendo análisis para conocer qué alimento le estamos suministrando a nuestros animales y estudiar la relación, nutrientes - suelo - planta - animal.

\section{Referencias}

BENAVIDES, E. 2004. Causas de muerte súbita en bovinos en pastoreo en las sabanas de América Tropical. Rev. Col. Cienc. Pecu. 17(2):182-192.

BENAVIDES, E.; LÓPEZ, M.; ALAYÓN, L. 2011. Enfermedades del ganado en la región de La Macarena (Meta). Un ejercicio de epidemiología participativa. Rev. Med. Vet. (21):41-62.

CAMPUZANO, G. 2011. Pica: el síntoma olvidado. Medicina \& Laboratorio. 17(11-12):533-552.

FIESTAS-TEQUE, L. 2014. Pica en anemia severa: a propósito de un caso. Rev. Neuro-Psiquiatría. 77(2):123-127.

FIRYAL, S. 2007. Pica (Depraved appetite; allotrophagia) in domestic animals. Pakistan Vet. J. 27 (4): 208-210.

GONZÁlEZ, S.; VALENCIA, A. 2011. Reporte de Caso: Indigestion Ruminal en un Bovino por elementos Indigestibles. Rev. Fac. Cienc. Agr. 3(1): 47-53.

HUND, A.; BEER, T.; WITTEK, T. 2016. Abomasal ulcers in slaughtered cattle in Austria. Tier. Prax. Gr. 44(5):279-85.

LOPES, A.; MACHADO, R.; DE TARSO LANDGRAF, P. 2013. Deficiência de cobre condicionada a altos teores de zinco, manganês e ferro na região do Médio Paraíba, RJ, Brasil. Semina: Ciênc. Ag. 34 (3): 1293-1300.

MALAFAIA, P.; NETO, J.; TOKARNIA, C.; OLIVEIRA, C. 2011. Distúrbios comportamentais em ruminantes não associados a doenças: origem, significado e importância. Pesq. Vet. Bras. 31(9):781-90.

MARTINS, A.; LEME, M.; PORTUGAL, M.; BALDASSI, L.; MARGATHO, L. 2004. Presença de corpos estranhos no habituais no aparelho digestório dos bovinos. Arq. Inst. Biol. 71(1):83-87.

MUZZI, L.; MUZZI, R.; GABELLINI, E. 2009. Técnica de fistulação e canulação do rúmen em bovinos e ovinos. Ciênc. agrotecnol. 33: 2059-2064.

OCAL, N.; GOKCE, G.; GUCU, A.; UZLU, E.; YAGCI, B.; URAL, K. 2008. Pica as a predisposing factor for traumatic reticuloperitonitis in dairy cattle: serum mineral concentrations and hematological findings. J. Anim. Vet. Advan. 7(6):651-656.

OLIVEIRA C, DE MATTOS F. 2010. Funções e sintomas de deficiência dos minerais essenciais utilizados para suplementação dos bovinos de corte. UNICiências. 14(2): 199-223.

ROSSANIGO, C.; FRASINELLI, C.; BENGOLEA, A. 2014. Pica en vacas en parición confinadas con silo de autoconsumo y agua de bebida de bajo tenor salino. Rev. Arg. Prod. Anim. 34(Supl 1):45-75.

SIMSEK, A.; SEKIN, S.; ICEN, H.; KOCHAN, A.; CELIK, O.; YAMAN, T. 2015. Abomasal impaction due to sand accumulation in two cows. Large An. Rev. 21(3):125-127. 